Meta

Journal des traducteurs

Translators' Journal

\title{
Présent et futur de la traduction publicitaire : entre eikôs et pathos
}

\section{Daniela Ventura}

Volume 54, numéro 3, septembre 2009

URI : https://id.erudit.org/iderudit/038308ar

DOI : https://doi.org/10.7202/038308ar

Aller au sommaire du numéro

\section{Éditeur(s)}

Les Presses de l'Université de Montréal

\section{ISSN}

0026-0452 (imprimé)

1492-1421 (numérique)

Découvrir la revue

Citer cet article

Ventura, D. (2009). Présent et futur de la traduction publicitaire : entre eikôs et pathos. Meta, 54(3), 450-465. https://doi.org/10.7202/038308ar

\section{Résumé de l'article}

En traduction publicitaire, la pratique consistant à laisser l'annonce inchangée, à savoir sans aucune modification textuelle ou iconique quels qu'en soient les destinataires et les marchés nationaux ciblés, est peu courante. Seuls certains produits échappent au processus de transformation. Le discours publicitaire étant persuasif par sa nature même, le traducteur se trouve forcé de répondre à la fois aux exigences du code de la langue et des lois de la rhétorique. La présente étude a pour objet l'analyse des stratégies de traduction adoptées en fonction de la typologie du produit commercial annoncé, de la visée argumentative et du destinataire. 


\title{
Présent et futur de la traduction publicitaire: entre eikôs et pathos
}

\author{
DANIELA VENTURA \\ Universidad de Las Palmas de Gran Canaria, Las Palmas de Gran Canaria, Espagne \\ dventura@dfm.ulpgc.es
}

\begin{abstract}
RÉSUMÉ
En traduction publicitaire, la pratique consistant à laisser l'annonce inchangée, à savoir sans aucune modification textuelle ou iconique quels qu'en soient les destinataires et les marchés nationaux ciblés, est peu courante. Seuls certains produits échappent au processus de transformation. Le discours publicitaire étant persuasif par sa nature même, le traducteur se trouve forcé de répondre à la fois aux exigences du code de la langue et des lois de la rhétorique. La présente étude a pour objet l'analyse des stratégies de traduction adoptées en fonction de la typologie du produit commercial annoncé, de la visée argumentative et du destinataire.
\end{abstract}

\begin{abstract}
Advertising language strategy for all markets and for all kinds of public is unusual. There are few products which do not need alteration in text or in image. Due to the persuasive nature of the advertising discourse, the duty of translators is to follow the rules of language code and rhetoric. The aim of this article is to analyze the translation strategies borrowed by translators according to the kind of product advertised, to the argumentative aim and to the addressee.
\end{abstract}

\section{MOTS-CLÉS/KEYWORDS}

adaptation, argumentation, eikôs, pathos, psychopublicité

adaptation, argumentation, eikôs, pathos, psycho-advertising

\section{Introduction}

En traduction publicitaire, la pratique consistant à laisser l'annonce inchangée, c'està-dire sans aucune modification textuelle ou iconique quels qu'en soient les destinataires et les marchés nationaux ciblés, est peu courante. Seuls certains produits échappent au processus de transformation: en effet, lorsqu'une marque doit avoir recours à une argumentation complexe, et que non seulement la langue mais aussi la culture font obstacle à une campagne de promotion internationale, la traduction, voire l'adaptation, s'imposent dans toutes leurs formes.

Dans l'intention de déterminer les tendances des pratiques actuelles de la traduction publicitaire et d'en déterminer la portée, nous avons examiné un corpus constitué d'une centaine d'annonces publicitaires parues dans la presse écrite de plusieurs pays et donc en différentes langues ${ }^{1}$. La présente étude, qui traite notamment des annonces françaises traduites en espagnol, a pour objet l'analyse des stratégies de traduction adoptées en fonction de la typologie du produit annoncé et de la visée argumentative. 


\section{L’annonce «mondialisée»}

Le fait qu'une annonce publicitaire ne subisse aucune transformation iconique ou textuelle dans une campagne de promotion internationale est déterminé par plusieurs paramètres, comme la nature intrinsèque du produit à promouvoir, sa renommée ainsi que les techniques de présentation. Il s’agit en général de produits tels que les articles de luxe (parfums, maroquinerie, vêtements, bijoux, etc.) et certains produits de grande consommation partout dans le monde (tabac, alcool, etc.) mis en marché par de grandes entreprises multinationales.

Dans le cas des articles de luxe, l'annonce joue avec l'atout de la beauté, la fascination, l'exotisme. Les Dior, Armani, Valentino, Prada, Yves Saint-Laurent, Louis Vuitton... - la liste serait interminable - ont recours à des annonces dont l'argumentation ne repose que sur la renommée de la marque. En ce qui concerne les produits de grande consommation, la marque ou le logo semblent se suffire à eux-mêmes. Coca-Cola, Marlboro et Nike, entre autres, ne semblent pas avoir besoin d'argumentation très développée pour atteindre leur public. Le slogan, s'il existe, n’est généralement pas traduit, surtout si la langue d'origine est l'anglais ${ }^{2}$. L'annonce d'une grande campagne de promotion internationale est conçue dès le départ pour être valable partout et accessible directement à tous les récepteurs, toutes cultures confondues ${ }^{3}$.

On peut distinguer deux types d'annonces reposant sur ce principe, selon qu'elles comportent ou non du texte. 1) Conçu dès le départ pour un marché mondial (et mondialisé), le premier type d'annonce se concentre essentiellement sur l'image, le nom de marque et/ou le logo occupant l'espace généralement consacré au texte. Il s'agit de ce qu'Adam et Bonhomme (1997) appellent une «annonce de fidélisation». Deux cas de figure se présentent. Dans le premier, l'annonce se sert de la beauté, de la sensualité ou de l'exotisme pour ajouter de la valeur (marchande) à une marque déjà connue et qui, pour vendre, n'a plus besoin de s'annoncer, au sens propre du terme. C'est en général le cas des produits de beauté, des parfums ou des voitures de luxe, pour lesquels la composante séductrice de l'argumentation est privilégiée: la photo d'un mannequin ou d'une actrice mondialement connus, à côté d'un sac à main et du nom de la marque, paraît suffisamment persuasive. Faisant appel aux distinctions de Grize (1981: 29), Adam et Bonhomme (1997) affirment que l'image publicitaire

[...] privilégie la composante 'séductrice' de l'argumentation. S'intégrant dans le MOVERE (émouvoir) et le PLACERE (plaire) de la rhétorique, exploitant par ailleurs des procédures floues axées sur les croyances et les jugements de valeur, l'argumentation par séduction vise à influencer son récepteur au moyen de stratégies affectives, davantage mobilisatrices que convaincantes (Adam et Bonhomme 1997: 194).

Le deuxième cas de figure concerne l'annonce qui cherche à toucher. D'après Perelman et Olbrechts-Tyteca (1992: 23), «[1]a plupart des formes de publicité et de propagande se préoccupent, avant tout, d'accrocher l'intérêt d'un public indifférent, condition indispensable pour la mise en œuvre de n'importe quelle argumentation ». C'est le I du sigle AIDA qui, d'après Boivineau (1972: 8), résumerait la formule magique du publicitaire: «A : attirer l'attention; I: susciter l'intérêt; D: éveiller le désir; A : provoquer l'achat». Pensons, par exemple, à Benetton, dont les annonces de vêtements, signées pendant une dizaine d'années par le photographe Toscani et particulièrement controversées, ne fonctionnent que par la force polémique d'une image 
touchante, voire choquante. Dans les deux cas de figure, que ce soit par la séduction ou par la provocation, ce genre d'annonces fait appel à l'émotion du public.

2) Le deuxième type d'annonce se compose d'un élément iconique auquel s'ajoute un élément textuel, le slogan, qui apparaît dans la langue originale ou en anglais (lingua franca). Des cas très particuliers, où les langues se mélangent, sont parfois observés. Ainsi, le parfum de Givenchy Fresh Attitude (The new fragrance) est annoncé dans le magazine espagnol Muy Interesante (mars 2007, $\mathrm{n}^{\circ} 310$ ) avec un slogan en franco-anglais: Very irrésistible Givenchy. Il s'agit encore une fois de marques mondialement connues qui, en général, proposent des produits dont la renommée se suffit à elle-même. Cela dit, il arrive parfois que certaines marques, dont la renommée ne fait aucun doute, recourent à la traduction. Pensons, entre autres, à Lancôme qui, malgré la simplicité du texte facilement compréhensible pour un lecteur espagnol, traduit littéralement le slogan de son parfum Trésor Le parfum des instants précieux: Trésor, el perfume de los instantes preciosos. Le slogan est présent, qu’il s'agisse d'une annonce de fidélisation ou du lancement d'un nouveau produit. Ainsi, Nike lance en Espagne ${ }^{4}$ ses nouvelles chaussures de sports Air Max 360 avec une simple photo représentant une chaussure suspendue dans les airs et accompagnée d'un slogan minimaliste en anglais, Run on air. Si le slogan n'a pas été traduit, c'est parce qu'il est censé être suffisamment clair et accessible à une large part du marché espagnol. Pour d'autres langues que l'anglais, l'accroche se fonde sur un certain exotisme de la langue étrangère. Ainsi, Lacoste propose une collection de montres ${ }^{5}$ avec le slogan Un peu d'air sur terre et le nouveau parfum de Jean Paul Gaultier ${ }^{6}$ est accompagné du slogan Pour l'un comme l'autre.

Pour toutes ces annonces, le principe fondamental est que le message est destiné à tous les publics, quelle que soit la nationalité et, par conséquent, qu'il doit être, sous l'angle culturel, le plus neutre et le plus dépouillé possible dès sa conception. La traduction se révèle donc, dans la plupart des cas, inutile. L'élément fondamental de persuasion est iconique et relève d'une stratégie essentiellement affective. Dans ce cas, la persuasion ne passe (pratiquement) pas par le raisonnement, mais par les sentiments.

\section{De l'altérité}

En guise de prémisse à cette section, il convient de clarifier notre terminologie. Nous concevons l'altérité comme le caractère de ce qui est autre, et plus précisément, lorsque nous parlons d'altérité en traduction, à l'instar de Cordonnier (1995, 2002), nous nous référons à la tendance à respecter la culture de départ dans le texte (annonce). Ce concept ne doit pas être confondu avec la traduction littérale, car pour respecter la culture de l'autre, il est possible qu'on soit obligé de recourir à l'explicitation.

La publicité de certains produits ayant une appellation d'origine contrôlée $(\mathrm{AOC})^{7}$, tels que les grands crus, les produits du terroir au sens le plus large du terme, ainsi que les produits soi-disant typiques d'un pays donné, cherche à les distinguer des produits des multinationales en jouant sur leur authenticité et sur leur caractère distinctif en rapport avec la clientèle de connaisseurs ou d'amateurs. Elle est conçue dès sa création pour souligner l'origine du produit, qui devient une valeur fondamentale garantissant un certain savoir-faire traditionnel. 
Ainsi, pour le champagne, produit typique de France, le principe fondamental est que ce genre de produit doit préserver et défendre son caractère unique et exclusif. Ce principe se manifeste, de façon universelle, par la mise en relief de la saveur du terroir et, éventuellement, par la conservation de la langue du pays, notamment s'il s'agit d'une annonce de fidélisation. L'argumentation de ce genre d'annonces est assez simple et repose sur une série de prémisses et de valeurs communes auxquelles adhère un destinataire spécifique et très ciblé ayant un pouvoir d'achat élevé et le goût du luxe, car comme le rappelle Grize (1981: 30), «[i]l est nécessaire qu’il [le locuteur] se fasse, parmi d'autres, une représentation de son auditeur. Non seulement des connaissances qu'il a, mais des valeurs auxquelles il adhère», des valeurs telles que l'authenticité, la fraîcheur, la tradition, la saveur ou le savoir-faire.

Si l'annonce est rédigée dans la langue du pays d'origine du produit, le texte est alors réduit à l'essentiel. C'est le cas de l'annonce du champagne Moët \& Chandon ${ }^{8}$ dont la phrase d'assise (base line ${ }^{9}$ ), L'esprit Moët et Chandon, a une fonction d'accroche: d'une part, elle charme, séduit, frappe ou au moins attire l'attention beaucoup plus que ne le ferait la langue maternelle du récepteur, l'exotisme et la séduction ayant une part importante dans l'effet persuasif global de l'annonce. D'autre part, elle le rassure au sujet des véritables origines du produit et, par conséquent, de sa qualité.

Si l'élément textuel est plus développé et s'il y a traduction, cette dernière se fait dans un esprit de dévoilement ${ }^{10}$ apparent car, en réalité, son but n'est pas celui d'éduquer à l'étrangeté, mais plutôt celui d'en rappeler certaines valeurs (généralement admises) par une série de topoï ou lieux communs ${ }^{11}$, à savoir des «stéréotype[s] conceptuel[s]» (Adam et Bonhomme 1997: 111 ${ }^{12}$ ) faisant partie du bagage culturel universel du consommateur ciblé et auxquels, nous l'avons dit, il est censé adhérer. Ce type d'annonce fait de son drapeau national et de son authenticité son principal argument. Les campagnes réalisées par les offices de tourisme des différents pays y ont largement recours. Dans la campagne de l'été $2005^{13}$ menée par l'Office hellénique du tourisme et destinée aux Français, la Grèce y est décrite comme une «terre mythique» où le chant des sirènes «vous plonge dans des eaux d'un bleu profond et vous fait danser jusqu'à l'aube sous le regard bienveillant de Dionysos», une terre où «les mythes sont toujours vivants». Dans sa campagne de février $2007^{14}$ destinée à la France, l'office du tourisme du Maroc évoque la ville de Fès, «berceau de la civilisation marocaine», sa médina millénaire «classée au Patrimoine mondial de l'Unesco » et son artisanat, «riche d'un savoir ancestral». La campagne de l'été 2007 vantant l'Italie à un public allemand ${ }^{15}$ joue sur une offre assez variée ne soulignant pas seulement l'attrait exercé par ses villes d'art, sa mer et sa nature, mais aussi sur ses thermes procurant le bien-être: la persuasion se déploie dans ce cas par la séduction.

Certaines marques internationalement connues suivent l'exemple des marques des produits d'appellation d'origine contrôlée ou typiques en appuyant leur argumentation sur un lieu commun lié au pays ou à la région d'origine du produit. C'est le cas de l'annonce du gin Beefeater, parue en 2007 dans un magazine espagnol ${ }^{16}$, qui présente un ensemble d'images consistant en un mélange de photos et de dessins, clairement connotées, évoquant le passé et le présent de Londres, ville identifiable grâce à ses monuments (notamment le Big Ben), à la Tamise et au panneau d'une rue, Oxford Street. D’autres éléments (un jeune homme portant un chapeau melon, une canne et une chemise imprimée avec le drapeau anglais, un voilier, un sous-marin - rappel des Beatles?) confèrent à l'annonce un air indiscutablement britannique. 
Aucun texte descriptif ou explicatif ne s'ajoute à la composante iconique, sauf le nom de la marque, la ville (London) et le slogan (Forever Cosmopolitan Since 1820) qui n'est d'ailleurs pas traduit. Un seul élément textuel, imposé par la loi, est rédigé en espagnol: il apparaît à la verticale sur le côté gauche de la page, rappelant qu'il faut consommer avec modération et indiquant la teneur en alcool du gin. Il est clair que l'intention du publicitaire est de montrer, grâce à l'image dont l'interprétation est loin d'être arbitraire, que ce produit est bel et bien britannique avec toutes les valeurs (ajoutées) que cela sous-tend. Conçue pour des étrangers, l'annonce fonde son argumentation sur des topiques éveillant des souvenirs ou des sentiments positifs. Une annonce de la marque italienne d'eau minérale San Pellegrino ${ }^{17}$ destinée au marché français repose sur un principe semblable. L'élément iconique est représenté par une photo en noir et blanc - qui rappelle certaines scènes des films du néo-réalisme italien - représentant une scène familiale: des jeunes gens sont attablés dans une cuisine. La table est mise. On ne voit distinctement que deux hommes bruns, dont l'un porte un tablier, l'air détendu et amusé. À l'arrière-plan, une fenêtre, des fleurs dans un pot, des ustensiles de cuisine, un gros pain de campagne et une bouteille d'eau minérale San Pellegrino. Un élément textuel, tenant lieu d'accroche ainsi que de guide de lecture, apparaît en surimpression à droite de la scène familiale: Buvez en version originale. La bouteille est représentée en couleur au bas de l'annonce, avec, en surimpression le slogan en français: Vivre en italien. L'argumentation se fonde sur deux valeurs sûres et largement admises: l'authentique (version originale) et la famille.

Dans ce genre d'annonce, l'élément iconique joue un rôle essentiel, en transmettant le gros du message, car, comme le faisait déjà remarquer Barthes,

[e]n publicité la signification de l'image est assurément intentionnelle: ce sont certains attributs du produit qui forment a priori le signifiés du message publicitaire et ces signifiés doivent être transmis aussi clairement que possible; si l'image contient des signes, on est donc certain qu'en publicité ces signes sont pleins, formés en vue de la meilleure lecture: l'image publicitaire est franche, ou du moins emphatique (Barthes 1964/1993: 1417).

Les signes pleins de l'image publicitaire déjà repérés et analysés par Barthes ${ }^{18}$, dans la célèbre annonce des pâtes Panzani, continuent d'être employés par les publicitaires d'aujourd'hui, et apparemment avec succès, si l'on en juge par la campagne de sauces italiennes pour pâtes Bertolli réalisée pour l'Allemagne en 2007 et parue dans le magazine Für Sie (7 août 2007). Tout comme dans l'annonce française des pâtes Panzani des années 1960, on y évoque les couleurs du drapeau italien en recourant au basilic, à l'ail et aux tomates. La traduction du texte en allemand, qui accompagne l'élément iconique, insiste sur le caractère traditionnel des sauces: Klassische Pasta Saucen für jeden $\mathrm{Tag}^{19}$.

Les signes culturels - tels que l'italianité dans les deux annonces qui nous occupent - ne sont pas ressentis comme un obstacle pour la traduction; transmettant des valeurs idéalisées de l'autre, partagées par le destinataire, ces topiques n'évoquent pas une altérité qui étonne et qui empêche la transmission du message. Au contraire, ils en rappellent une facette procurant le bonheur, le bien-être ou, tout simplement, des souvenirs agréables, ce qui suscite l'adhésion du public visé. 


\section{Fidélité ou recréation?}

Que dire de la fidélité en publicité? D’après Tatilon (1990: 81), «le problème épineux de la fidélité ne se pose plus [...] il suffit de recréer», la consigne étant «traduire non la lettre mais l'esprit, non les mots mais les fonctions» (Tatilon 1990: 245). La traduction publicitaire est-elle donc toujours censée être "relâchée», "réduite à l'essentiel » (Tatilon 1990: 245)? Bien que nous soyons d'accord avec l'auteur sur le principe général, il nous paraît prudent de nuancer cette assertion dans la mesure où tout dépend des codes de langue en jeu et du type de discours publicitaire mobilisé. S'il s'agit de deux langues romanes, telles que l'espagnol et le français, et lorsque le texte de l'annonce est purement dénotatif (ce qui se produit souvent dans les annonces dont l'élément textuel est du genre pseudo-scientifique), on remarque un grand parallélisme entre le texte de départ et le texte d'arrivée. Cela est dû à l'importance que suppose, dans ce genre de texte, la fonction référentielle, d'où la nécessité de restituer le texte source en recourant à une terminologie exacte. Cela implique forcément un certain respect, toujours dans la mesure des possibilités offertes par le code de la langue d'arrivée, du genre dans lequel s'inscrit l'élément textuel de l'annonce. Voyons, à titre d'exemple, l'annonce de la crème Absolue premium Bx de Lancôme, dont le texte en français ainsi que la version espagnole sont présentés ci-dessous:

Nouveau ABSOLUE PREMIUM Bx / CRĖME RECONSTITUANTE PROFONDE IP 15 / RÉPARATION - NUTRITION - ÉCLAT

Sous l'effet de l'âge et des carences hormonales, la matrice fondamentale de la peau se dégrade. Lancôme invente le système anti-âge du $21^{\mathrm{e}}$ siècle en associant:

$>$ Le Pro-Xylane ${ }^{\mathrm{TM}}$, découverte scientifique majeure: la molécule anti-âge la plus puissante de notre recherche, pour reconstruire la matrice fondamentale de la peau à tous les niveaux*.

$>$ Le Bio-réseau reconstructeur ${ }^{\mathrm{TM}}$ enrichi - wild yam, soja, algue brune et orge - pour relancer les fonctions vitales de la peau.

Performances renforcées pour des résultats spectaculaires ${ }^{\star *}$ :

- Réparation: la peau est plus ferme (99\%), plus élastique (98\%).

- Nutrition: la peau est intensément nourrie, flexible, confortable (100\%).

- Éclat: la peau est éclatante (97\%), gorgée de vie!

(Santé magazine, $\mathrm{n}^{\circ} 374$, février 2004)

Nuevo ABSOLUE PREMIUM Bx / CREMA RECONSTITUYENTE PROFUNDA IP 15 / RECONSTRUCCIÓN - NUTRICIÓN - LUMINOSIDAD

Como consecuencia de la edad y de las carencias hormonales, la matriz fundamental de la piel se degrada. Con ABSOLUE PREMIUM Bx, Lancôme inventa el sistema antiedad ABSOLUE ${ }^{\mathrm{TM}}$ del siglo XXI mediante la asociación de:

$>$ El Pro-Xylane ${ }^{\mathrm{TM}}$, un descubrimiento científico revolucionario: La molécula antiedad más potente y más completa de nuestra investigación, para reconstruir la matriz fundamental de la piel en todos los niveles*.

$>$ La Bio-estructura reconstructora ${ }^{\mathrm{TM}}$ enriquecida - wild yam, soja, alga marrón y cebada - para relanzar las funciones vitales de la piel.

Eficacia con resultados espectaculares ${ }^{* *}$ :

Reconstrucción : la piel está más firme (99\%), más elástica (98\%), las arrugas se reducen.

Nutrición : la piel está intensamente nutrida (100\%), flexible y confortable (100\%).

Luminosidad: la piel está resplandeciente $(97 \%)$, ;rebosante de vida!

(El País semanal, mars 2007) 
Tout en gardant la fonctionnalité du texte-source, le traducteur a respecté le contenu et la forme pseudo-scientifique de l'original, le texte évoquant, dans ce cas concret, des notions partagées dans les deux langues. Dans certaines annonces de type narratif, on peut voir parfois une équivalence à la fois pragmatique et formelle entre le texte de départ et celui d'arrivée. Voyons, à titre d'exemple, les versions française et espagnole de l'annonce de la crème anti-rides de Clarins:

Un matin on se réveille. On a 40 ans. Et ça ne se voit pas. Ce matin, vous vous faites un cadeau: des années de jeunesse en plus. Grâce aux actifs anti-âge des soins MultiRégénérants de Clarins, votre visage garde tout son éclat et sa fermeté pour longtemps. Bon anniversaire. C’est prouvé. Clarins rend la vie plus belle.

(Elle, le 23 octobre 2006)

Una mañana nos despertamos. Tenemos 40 años. Y no se nota. Esa mañana usted se hace un regalo: unos años más de juventud. Gracias a los principios activos antienvejecimiento de los Tratamientos Multi-Regenerantes de Clarins, su rostro mantiene su resplandor y firmeza durante mucho tiempo. ¡Feliz cumpleaños! Es cierto. Con Clarins, la vida es más bella.

(Magazine, le 5 novembre 2006)

Exception faite pour la phrase d'assise ${ }^{20}$, C'est prouvé. Clarins rend la vie plus belle, le traducteur n'a pas dû recourir à l'adaptation étant donné la nature du texte original. Que ce soit sur le plan du mot (signifiant) ou de la phrase, le texte d'arrivée se moule sur celui de départ. Il en résulte un texte à la fois correct et idiomatique. Certes, on pourrait observer que la césure phrastique semble quelque peu forcée: Una mañana nos despertamos. Tenemos 40 años. Y no se nota. Dans l'usage normatif et naturel de la langue, on aurait plutôt uni les deux premières phrases par une virgule et la troisième avec la conjonction, tout simplement; cela dit, il est vrai aussi que la césure phrastique, telle qu'elle apparaît dans l'annonce originale, n'est pas plus usuelle en français qu'en espagnol. Aussi, nous semble-t-il, l'effet perlocutoire recherché dans le texte de départ est-il respecté dans le texte d'arrivée.

Les deux exemples d'annonces que nous venons d'analyser, tout en étant assez représentatifs d'une part considérable du marché, ne sont cependant pas légion, car cette équivalence entre les deux codes n'est guère automatique, la correspondance entre les deux langues n'étant souvent qu'apparente, et ce, même dans des textes où prédomine l'élément dénotatif.

\section{De la modulation à l'adaptation}

Si l'équivalence formelle entre le français et l'espagnol peut se révéler à la fois correcte et pragmatique dans les cas que nous venons de décrire, il n'en reste pas moins que même lorsque la nature du texte-source se prête à la correspondance, les défauts grossiers de traduction résultant d'un moulage excessif sur la langue de départ et entraînant faux sens et tournures forcées, sont plutôt fréquents, si bien que le message perd en efficacité.

Dans l'annonce de Lancôme d'un produit autobronzant (Flash bronzer), qui comporte cependant un texte à forte dominante dénotative, l'équivalence formelle n'est pas toujours possible ni souhaitable en espagnol, d'où la recherche d'une adaptation stylistique permettant de respecter, autant que possible, le registre argumentatif et les tournures usuelles de la langue d'arrivée. 
Nouveau

Sublimateur jambes

effet bronzé, nacré immédiat

Nouveau

Gel visage application record

TEMPS RECORD

POUR HÂLE PARFAIT

Demandez conseil à votre parfumeur agrégé

(Elle, mai 2005)
Nuevo

Realza las piernas con

un bronceado dorado inmediato

Nuevo

Gel rostro resultado récord TIEMPO RÉCORD

PARA UN BRONCEADO PERFECTO

(Magazine, juin 2005)

On se heurte ici à deux problèmes de fond. Le premier concerne le néologisme sublimateur qui aurait pu, certes, être restitué directement par son équivalent sublimador. Or, cette solution n'est pas souhaitable, non seulement parce que le terme n'est pas usuel en espagnol, mais aussi et surtout si l'on analyse la suite du texte-source qui se caractérise par son style télégraphique. Un tel style serait perçu comme très forcé en espagnol, car cette langue préfère une construction recourant aux verbes et aux prépositions, d'où le recours à une modulation libre qui, en paraphrasant Vinay et Darbelnet (1977: 51), aboutit à une solution qui fait s'exclamer le lecteur: «Oui, c'est bien comme cela que l'on s'exprimerait en espagnol.» On se heurte sur le plan référentiel au deuxième problème: si le mot nacré était traduit par (a)nacarado, celuici renverrait dans ce contexte à une référence culturellement presque opposée à celle évoquée par le français. Les reflets irisés de la nacre, quoique très poétiques, ne correspondent pas à l'idée qu'un hispanophone peut se faire de la peau bronzée. En effet, l'adjectif (a)nacarado renvoie plutôt, dans l'imaginaire collectif, et dans ce cas concret, à une couleur blanche brillante, éclatante ${ }^{21}$, d'où le choix, fonctionnel et opératoire, de dorado (/doré/), le procédé de l'adaptation étant non seulement souhaitable mais nécessaire. Il faut souligner que l'élément iconique, constitué par une photo d'Inés Sastre, une top-modèle espagnole très bronzée et dont les jambes occupent une position de tout premier plan, joue un rôle d'exemple et fonctionne comme référence absolue du rédactionnel. Enfin, la version espagnole comporte certaines omissions: elle n'a pas retenu d'équivalent du mot effet (effet bronzé) et semble préférer à l'illusion de l'effet bronzé la réalité du bronceado. Une autre omission se réalise par l'absence de traduction du syntagme: Demandez conseil à votre parfumeur agréé. D’ailleurs, l'omission de cet élément textuel se répète presque systématiquement dans nombre d'annonces de produits de beauté de certaines maisons françaises telles que Roc et Vichy (Demandez conseil à votre pharmacien), l'Oréal (Besoin de conseils?) ou encore Liérac (Demandez conseil à votre pharmacien ou à Liérac), entre autres. Cette absence systématique peut répondre à des normes moins strictes en Espagne qu'en France quoique, la raison essentielle, nous semble-t-il, repose sur un comportement généralement admis en Espagne quant à l'automédication.

Le recours à l'adaptation est particulièrement manifeste pour le slogan ou la phrase d'assise. Si un slogan tel que celui des Laboratoires Forté Pharma (Laboratoires Forté Pharma, donner plus de vie à la vie) ne suppose pas un grand effort d'adaptation en espagnol (Laboratoires Forté Pharma, da más vida a la vida), cela n’est pas vrai dans la plupart des cas, notamment lorsqu'il est question de jeux de mots, de double sens ou de calembours, monnaie courante dans les annonces en français. Pensons à la phrase d'assise de l'annonce de la Renault Espace: Et si le vrai luxe c'était l'Espace? Le jeu de mots entre le nom de la voiture, Espace et le substantif espace n'étant pas 
transposable en espagnol; le traducteur a néanmoins tenu compte de la thèse à illustrer, autrement dit, que la voiture est spacieuse: ¿Y si el verdadero lujo fuera el espacio? Toutefois, il est possible que la minuscule du nom commun espacio n'évoque pas d'emblée, chez le lecteur non avisé, l'association avec le nom propre Espace, association qu'il sera quand même en mesure de faire après lecture du texte explicatif accompagnant la phrase d'assise et la photo de l'intérieur de la voiture. Ce choix du traducteur ne nuit aucunement à la stratégie persuasive du message publicitaire, compte tenu de l'apport non indifférent du support iconique zoomant sur les sièges de la voiture.

Le slogan, réduit souvent au minimum, peut fonctionner dans une langue de façon perlocutoire, tandis que sa traduction directe ne fonctionnera pas du tout dans une autre, d'où la décision du traducteur de l'adapter pour rendre compte clairement et de manière justifiée d'une stratégie persuasive. Ce cas de figure se produit notamment lorsque, afin d'atteindre le destinataire, le slogan ou la phrase d'assise se déguise sciemment en ce qu'Adam et Bonhomme (1997: 37) nomment dialogisme feint, soit « [u]n échange illusoire [entre annonceur et consommateur], canalisé par l'annonceur, qui ne permet aucune réponse véritable et ne change rien à la distribution des places d'émetteur et de destinataire». Dans le but de persuader, ce dialogisme, quoique feint, se doit cependant d'être transparent. Plusieurs possibilités de traduction sont alors envisageables. La traduction du vouvoiement de politesse est un problème fréquent, résolu de façon variable et assez libre: certains traduisent littéralement par usted, tandis que d'autres adoptent le tutoiement. Lorsque l'impératif est utilisé, le traducteur peut choisir l'infinitif, notamment quand il s'agit de donner des conseils: par exemple, dans l'annonce d'un complément nutritionnel des Laboratoires Forté Pharma, Aspirez / Drainez (Elle, le 18 avril 2005) s'est transformé en Aspirar / Drenar (Pronto, le 22 avril 2006). Pour le célèbre slogan de L’Oréal, Parce que vous le valez bien, le traducteur a préféré le tutoiement: Porque tú lo vales. La littéralité, qui impliquerait, en espagnol, l'emploi de la troisième personne de politesse usted pour rendre le vous français aurait sans doute été possible et, grammaticalement, voire sémantiquement, correcte. Cependant, le vouvoiement en espagnol peut produire un sentiment désagréable chez le lecteur espagnol, cette forme de politesse étant généralement réservée à une personne âgée, d'où l'emploi de plus en plus fréquent $\mathrm{du}$ tutoiement, parfois déplacé, pour insister sur l'apparence juvénile d'une personne, surtout lorsqu'il s'agit d'une femme, notamment dans les grandes surfaces et les boutiques en général. Le tutoiement, compte tenu de la catégorie de produits vendus par L'Oréal (notamment les crèmes anti-âge ${ }^{22}$ ), paraît donc plus fonctionnel que le vouvoiement.

Tout en gardant la visée persuasive du texte-source, le slogan de la Peugeot 1007 (campagne de 2005), Trop facile!, est amplifié et modulé en une phrase affirmative plus complexe, Te facilita la vida (Elle vous rend la vie facile), qui se présente sous forme de dialogisme feint.

Afin d'éviter une adhésion trop stricte au texte-source en faveur d'un texte d'arrivée acceptable et notamment persuasif dans la langue cible, l'équivalence dynamique (Nida 1964) s'impose. La tendance marquée, en traduction publicitaire, au recours à l'adaptation répond en partie aux exigences du code, mais surtout aux lois de la rhétorique, car, ne l'oublions pas, «le discours publicitaire est un discours de la persuasion» (Tatilon 1990: 243). 


\section{De l'adaptation à la transposition culturelle}

La théorie selon laquelle il faut traduire non la lettre, mais l'esprit et gommer les aspérités culturelles constituant la pierre d'achoppement d'un texte trouve une application exceptionnellement féconde en traduction publicitaire. Ladaptation du message et la prise en compte des attentes culturelles du récepteur sont la règle dans le domaine de la publicité, qui suit une pratique ethnocentrique de la traduction, soit une tendance à privilégier le groupe social auquel on appartient et à en faire le seul modèle de référence (Cordonnier 1995). Comme le souligne Boivineau (1972: 12), l'adaptateur «[d]evra faire disparaître tout ce que la publicité comporte d'étranger au milieu auquel il s'adresse, en s'identifiant parfaitement à ce milieu, en le connaissant à fond». Il s'agit donc de réaliser une transposition culturelle ayant pour objectif de polir, voire effacer, les écueils culturels contenus dans l'annonce originale. Cependant, cette pratique ne relève pas véritablement d'une théorie de la traduction proprement dite, mais plutôt, essentiellement, de la nature argumentative du discours publicitaire qui impose que l'on s'adapte à son public. À l'instar d'Aristote, Perelman et Olbrechts-Tyteca (1988: 24) rappellent que pour que l'argumentation soit efficace, il faut connaître ceux que l'on se propose de convaincre. C'est pourquoi, forts des études de marché, des enquêtes de consommateurs ainsi que des recherches ad hoc menées par des psychologues, les publicitaires étudient à la loupe leur public et ses attentes.

Le traducteur, lui, se trouve donc forcé de répondre à la fois aux exigences du code et aux lois de la rhétorique. Le besoin impérieux de s'adapter au public fait en sorte que tout élément connoté (donc hermétique) dans le texte-source soit nécessairement passé au crible et restitué de manière claire et intelligible (donc persuasive) dans le texte d'arrivée, tâche particulièrement difficile, car il faut tout d'abord savoir distinguer ce qui est connoté de ce qui ne l'est pas. En effet, comme le souligne Kerbrat-Orecchioni (1977: 13), « [le] principal problème que pose la connotation à l'analyse linguistique, c'est celui de définir la ligne de démarcation qui sépare dénotation de connotation [...].» Et Ladmiral (1994: 168) de renchérir: «[i]l est extrêmement difficile, sinon même impossible, de faire le départ entre dénotations et connotations", ce qui constitue donc une difficulté de plus à surmonter lors de la traduction publicitaire.

Voyons, en guise d'exemple, l'annonce d'un produit de beauté des laboratoires Payot qui lancent, en 1998, une gamme cosmétique de crèmes revitalisantes. La campagne publicitaire se renouvelle en 2005, avec cinq nouveaux produits et de nouvelles formulations. Le texte original en français et la traduction en espagnol sont indiqués ci-dessous (les mots en italique se trouvent dans l'original et dans la version):

Vivre sans stress par Léa Bancourt. 1. Rentrer plus tôt. 2. Une douche chaude, mon peignoir. 3. Un peu de musique et un dîner léger. 4. Crème de Rêves Payot, pour que ma peau aussi se repose. 5. Bien dormir, faire de beaux rêves. 6 . Se réveiller doucement, avec plaisir. 7. Crème de Choc Payot, pour réveiller l'éclat de ma peau. 8. Un pamplemousse pressé, les nouvelles et c'est parti!

(Elle, 2000)

Vivir sin estrés por Léa Bancourt. 1. Llegar pronto a casa. 2. Ducha caliente. Pijama. 3. Un poco de música y una cena ligera. 4. Crème de Rêves Payot, para que mi piel 
descanse. 5. Dormir bien. Dulces sueños. 6. Despertar lentamente. Sin prisas, 7. Crème de Choc Payot, para que mi piel despierte llena de energía. 8. Zumo de naranja, las noticias y ... ¡Lista!

(El País semanal, 2001)

Une omission est repérable d'emblée, l'idée de /plus/: rentrer plus tôt est rendue par llegar pronto a casa (/rentrer tôt/), ce qui implique déjà une certaine liberté dans la traduction. Bien qu'on puisse attribuer l'omission à un simple oubli du traducteur, nous croyons plutôt qu'elle est voulue. Les horaires de travail des Espagnols et des Français sont remarquablement différents. Il est en effet inimaginable pour un Espagnol de terminer sa journée à dix-sept heures, heure à laquelle, de façon générale, il commence probablement son après-midi. Afin que le destinataire espagnol adhère au message, le traducteur a senti donc l'obligation d'omettre cette idée de /plus/, omission à notre sens réussie sur le plan perlocutoire, car tout en changeant inexorablement le point de vue (les horaires de référence ne sont guère les mêmes dans l'esprit des Espagnols et des Français), elle permet de garder la crédibilité de l'argumentation. Il faut souligner, par ailleurs, la métamorphose du peignoir en pijama (/pyjama/) et du pamplemousse en naranja (/orange/). Ainsi se manifeste une transposition culturelle s'expliquant par le fait que la traduction publicitaire se moule, comme l'affirment Bonhomme et Rinn (1997: 15), "sur l'aire culturelle associée à chaque langue, de façon à accroître sa crédibilité et son intérêt positif en favorisant l'adhésion des groupes sociolinguistiques qu'elle cible: plus le message se plie à cette loi contextuelle de proximité [...] plus sa force persuasive est garantie». En effet, l'orange et le pyjama sont beaucoup plus caractéristiques de la culture cible que le pamplemousse et le peignoir, ce qui renforce le caractère persuasif de l'annonce. Il est possible que le choix de l'orange, clairement ethnocentrique, soit dû au moins à deux raisons de poids: d'une part, les Espagnols n'ont pas l'habitude de boire du jus de pamplemousse et encore moins le matin au petit-déjeuner; d'autre part, l'Espagne est le pays des oranges, d'où sa consommation à grande échelle. Le choix de l'idée du pyjama est également justifiable culturellement: pour traduire le mot peignoir, on aurait pu utiliser albornoz ou bata. Bata a plusieurs sens et emplois. Fortement connoté, bata aurait été culturellement associé, dans ce contexte, à une image nonchalante de la femme au foyer, ce qui explique son rejet, n'étant pas fonctionnel. Albornoz n'est pas fonctionnel non plus, surtout en rapport avec les horaires de travail des Espagnols. Si l'on rentre chez soi vers six heures du soir, voire plus tôt, comme c'est le cas en France et comme l'envisage l'annonce dans sa version originale, il est compréhensible que l'on mette son peignoir. Si l'on rentre vers vingt et une heures, il est en revanche plus vraisemblable d'enfiler son pyjama après une douche. Selon Boivineau (1972),

l'adaptateur devra veiller à éliminer de l'annonce tout ce qui peut choquer le consommateur dans ses croyances, ses sentiments - y compris le sentiment national, - ses traditions, ses attitudes, ses coutumes, son comportement, autrement dit dans tout ce qui constitue son patrimoine culturel (Boivineau 1972:12).

Le traducteur a ainsi évité le choc culturel en naturalisant la France, terme que nous empruntons à Lederer (1994: 126) selon laquelle «le souci de faire accepter l'autre va parfois au-delà de la préoccupation de le faire connaître et il arrive que le traducteur substitue des faits de sa propre culture à ceux qu'évoque le texte; il les naturalise». 
Si, selon nous, cela est tout à fait répréhensible dans la traduction d'un texte littéraire, cette attitude s'explique en traduction publicitaire et se justifie étant donné la visée persuasive du discours publicitaire et un certain pragmatisme commercial. Comme le rappellent Bonhomme et Rinn (1977: 11), on ne traduit pas seulement un code mais également «une production énonciative complexe, avec tout l'univers qui la sous-tend». Cette annonce de Payot constitue en ce sens l'exemple typique de la pratique ethnocentrique de la traduction publicitaire. Issue du moulage sur l'aire culturelle liée à l'Espagne, la version modulée de l'annonce française est sans doute plus fonctionnelle que l'équivalence formelle. Le résultat obtenu pourrait être défini, selon les termes de Boivineau (1972: 15), comme une Belle efficace.

\section{Du principe de vraisemblance}

Revenons en arrière. Compte tenu de la nature du discours publicitaire, et dans le but d'obtenir l'adhésion de son public pour persuader, le traducteur publicitaire doit se soumettre à une multiplicité de contraintes qui rendent sa tâche peu aisée. Il fera son choix en fonction du type du texte, de son idiolecte et de la ligne argumentative de la marque. Il n'a d'ailleurs pas toujours les coudées franches, celle-ci imposant souvent des normes très strictes quant à la reformulation du texte de départ. Par conséquent, un texte-source peut produire une infinité de versions plus ou moins fonctionnelles dans la langue d'arrivée.

Notre analyse a montré que certaines maisons, de renommée internationale, conçoivent leurs campagnes avec une visée mondiale, en misant sur la neutralité d'un message argumentatif s'adressant ainsi à une multiplicité de publics et de cultures. Cependant, chez les multinationales, la tendance récente se trouve être inversée, car elles commencent à diversifier leur image en fonction des marchés nationaux. Ainsi, L'Oréal a choisi trois ambassadrices différentes: Laetitia Casta pour l'Europe, Aishwarya Rai pour l'Inde et Gong Li pour la Chine. Même Coca-Cola chercherait à adapter ses publicités en fonction de la culture cible. C'est ce que Guy Cook nomme copy adaptation: "The procedure known as copy adaptation can result in an extreme case of translation, a form of cultural transposition, which involves the production of a totally different advert or campaign for each of the countries in which a product is launched or promoted» (Cook cité dans de Pedro 1996: 29-31).

À côté des annonces mondialisées, rédigées en anglais ou dans la langue originale, il existe toujours un certain nombre d'annonces pour lesquelles la traduction joue un rôle capital. L'équivalence formelle occupe encore une place considérable en traduction publicitaire, notamment lorsqu'il est question de codes appartenant à une même famille et en présence de textes essentiellement dénotatifs. Force est de constater, toutefois, que si la littéralité peut se révéler, dans certains cas, opératoire, elle entraîne souvent une série de risques sur le plan sémantique, car aboutissant à un texte d'arrivée dont le registre argumentatif est totalement déplacé: d'où sa perte de fonctionnalité et le besoin d'adapter, voire de réécrire, le texte-source, et cela de deux façons possibles: la première consiste à valoriser l'Autre et la seconde, à l'effaçer. Le premier cas de figure s'applique notamment aux annonces des produits typiques (cas des AOC) ou prétendus tels. L'argumentation ne cherche pas à s'adapter à la culture du destinataire: au contraire, elle dévoile la culture d'origine, ou, pour mieux dire, certaines facettes (topiques) positives de la culture d'origine que le destinataire est censé connaître. 
À l'opposé, dans la forme mais pas dans le but final, se trouve l'adaptation qui gomme les aspérités culturelles, vécues comme un obstacle majeur de la communication (et donc des ventes), en les naturalisant. Cette tendance concerne la plupart des annonces de produits existant sur le marché. Considérée par Guidère (2001) comme une fonction commerciale dont la mission consiste à adapter les stratégies commerciales aux groupes de marchés nationaux, la traduction publicitaire n'aime pas montrer la culture d'origine. C'est pourquoi, dans les campagnes de promotion à l'échelle internationale, l'annonce dont l'élément argumentatif est important souffre généralement d'une adaptation opérant une transposition culturelle en fonction du pays ciblé. Cette pratique ethnocentrique de la traduction s'explique du fait que si les prémisses culturelles dont part l'argumentation publicitaire ne jouissent pas de l'adhésion du destinataire, comme nous le disions plus haut, le message perd en fonctionnalité. Pour atteindre le destinataire et pour être persuasive, l'argumentation de l'annonce peut se fonder sur des vérités admises, mais aussi sur des présomptions liées à ce qui arrive normalement, le fréquent (eikôs) et donc en rapport avec l'expérience commune et l'opinion de la majorité (doxa $)^{23}$, car, comme disait déjà Platon (1974), la persuasion relève de la vraisemblance. Le traducteur se doit alors de respecter ce principe de vraisemblance pour éviter le choc culturel pouvant provoquer du désagrément chez le consommateur et, par conséquent, le rejet du message. Soulignons cependant que les présomptions servant à bâtir les argumentations peuvent être aussi contredites par les faits, car l'inattendu n'est pas à exclure (Perelman 1977). Selon Tatilon (1990: 243), l'inattendu serait même l'un des «principaux attributs» de la publicité, ce qui expliquerait depuis un certain temps la naissance d'une publicité nouvelle cherchant à éveiller l'attention du consommateur en le provoquant avec des images étranges, choquantes. Cela dit, si l'on admet avec Charaudeau (2004: 35) que l'étrangeté «attire et donne envie d'entrer en relation avec l'autre», il est tout aussi vrai que «[l']attirance se transforme [...] en mouvement de rejet ou d'inquiétude», d'où la tendance généralisée, dans le passé et de manière générale encore de nos jours, en publicité et en traduction publicitaire à miser sur les valeurs sûres du socialement admis et de l'opinion commune.

\section{Entre aujourd'hui et demain : à la recherche des émotions}

Le monde de la publicité change à une vitesse vertigineuse grâce aux progrès des neurosciences qui permettent désormais de déterminer comment réagit le cerveau face à certains stimuli. Zuberoa (2007: 41) remarque que la neuroéconomie produit une révolution dans le marketing et en publicité «en manipulant le cerveau du consommateur afin de transformer le produit en un désir mental auquel personne ne peut résister» (traduction libre).

De nos jours, la publicité classique commence à perdre du terrain. L'annonce fondant son argumentation sur la qualité et le prix du produit attire de moins en moins l'attention du consommateur désormais friand de nouveautés. Les professionnels de la publicité prennent de plus en plus conscience du fait que même les valeurs sûres, psychologiquement rassurantes, finissent, à la longue, par lasser. Alors que le neuromarketing aux États-Unis commence à faire fureur, les portes commencent déjà à s'ouvrir tout grand à la psychopublicité vendeuse d'émotions. Tout va désor- 
mais se jouer sur la manipulation du cerveau, le grand enjeu étant la recherche des émotions, facteur clé pour obtenir la persuasion.

Compte tenu de ces avancées dans l'univers du marketing international, le traducteur publicitaire devra-t-il réviser ses copies et s'adapter aux nouvelles stratégies de vente? Oui, sans doute, quoique, dans le fond, il n'y ait rien de nouveau sous le soleil de la persuasion, car la psychopublicité, loin d'être une découverte révolutionnaire, ne suppose qu'une confirmation scientifique des plus grandes et éternellement valables lois de la rhétorique. Pour Aristote (trad. 1991: 23), « [1]a persuasion est produite par la disposition des auditeurs, quand le discours les amène à éprouver une passion.» En effet, la persuasion ne se fait pas que par le biais de la démonstration, l'argumentation par le cheminement des émotions étant, sinon convaincante, au moins très mobilisatrice. Preti (1974: 60-61) remarque que la persuasion s'adresse à la partie irrationnelle (émotionnelle), subjective, de l'esprit humain. Selon Adam et Bonhomme (1997: 194), «l'argumentation par séduction vise à influencer son récepteur au moyen de stratégies affectives, davantage mobilisatrices que convaincantes». Et les auteurs de citer, en guise d'exemple, le cas des annonces de la marque Benetton signées par Toscani, dont «l'apparente anti-séduction [...] joue pleinement sur l'émotion» (Adam et Bonhomme 1997: 194), sur le pathos, d'où son extrême actualité et sa valeur exemplaire pour les prochaines générations de publicitaires. Compte tenu des progrès des neurosciences appliquées au marketing, nous pourrions désormais dire, en paraphrasant Aristote, qu'en publicité, la persuasion est produite par la disposition des consommateurs en puissance, quand le discours les amène à éprouver une émotion mentale.

Il reste à savoir si les émotions qu'on veut vendre sont universellement valables ou si, au contraire, elles sont culturellement prédéfinies. C’est sur cette réponse que se jouera le futur ou le déclin de la traduction publicitaire.

\section{NOTES}

1. Pour avoir une vision d'ensemble du marché européen de la traduction publicitaire sur une période assez longue, nous avons colligé et analysé, depuis 1999, des traductions d'annonces en espagnol, français, italien, anglais et allemand. Les annonces françaises analysées ont été tirées de Science et vie, Ça m’intéresse, Elle, Santé magazine, Marie Claire, Géo et Le Nouvel Observateur; les annonces espagnoles sont parues dans Elle (Espagne), Muy interesante, Magazine, El País semanal, Pronto, Mía, El Mundo, Geo et National Geographic (Espagne).

2. Dans les publications françaises, il arrive parfois qu'il soit traduit avec un astérisque. Signalons, par exemple, la marque SONY: Go create $=\grave{A}$ vous de créer.

3. Luciano Benetton déclarait que «La pub n'est pas faite pour vendre plus. Il s'agit de pub institutionnelle réalisée pour communiquer les valeurs de l'entreprise. [...] Nous avons besoin d'une image unique qui puisse passer de la même façon dans le monde entier» (Benetton 2006).

4. Muy interesante, $\mathrm{n}^{\circ} 297$, février 2006.

5. Revista de El Corte Inglés, hiver 2005.

6. Muy interesante, $\mathrm{n}^{\circ} 308$, janvier 2007.

7. «Lappellation d'origine contrôlée (AOC) désigne un produit originaire d'une région ou d'un lieu déterminé, dont les caractéristiques sont dues essentiellement à ce milieu géographique. Elle résulte de la combinaison d'une production et d'un terroir qui s'exprime par le savoir-faire des hommes » (Ministère français de l'agriculture et de la pêche 2007).

8. National Geographic (édition espagnole), décembre 1999.

9. Les deux termes sont employés par Adam et Bonhomme (1997: 59).

10. Terme emprunté à Cordonnier $(1995,2002)$.

11. "Aristote les appelle "communs" non seulement parce qu'ils dépassent le cadre de tout type de discours, ce sont des universels formels, mais aussi parce que ce sont les modes dont tous les 
hommes constituent en général leurs raisonnements, y compris ceux qui le feraient d'une manière toute intuitive» (Vega y Vega 2000: 133).

12. Adam et Bonhomme (1997: 111) entendent le topos comme «un stéréotype conceptuel d'ordre logico-discursif et fondé sur l'opinion commune (la doxa) qui permet de jeter un pont entre une donnée et une conclusion».

13. Géo, juin 2005.

14. L’Express, 22 février 2007.

15. Freundin, 14 août 2007.

16. Muy interesante, mars 2007.

17. L'Express, le 28 juin 2007.

18. Nous renvoyons le lecteur au célèbre article de Roland Barthes «Rhétorique de l’image» (1964/1993) dans lequel il analyse du point de vue dénotatif et connotatif l'image d'une annonce de pâtes de la marque Panzani. L'image est composée d'un paquet de pâtes - dont on remarque clairement la marque et les couleurs (vert, blanc et rouge) -, d'une tomate, d'un champignon, d'oignons, d'un poivron, d'une petite boîte et d'un filet blanc, le tout sur un fond rouge. En bas, le texte en blanc et en majuscules: PÂTES - SAUCE - PARMESAN. À L'ITALIENNE DE LUXE.

19. «Les sauces classiques pour pâtes Bertolli vont vous ravir chaque jour.» Nous adaptons. Le mot für est en italique dans le texte original.

20. Nous croyons avec Ladmiral (2004:23) qu' «'il n'existe pas de point où s'arrête la traduction et où commence l'adaptation". En fait, c'est là l'énoncé d'un problème fondamental. En un mot: je dirai qu'on a affaire à un continuum. C'est-à-dire qu'on va progressivement, et insensiblement, d'un extrême à l'autre.»

21. Dans le Diccionario de la Lengua Española (2005), on définit le mot nácar comme une «Sustancia dura, blanca, brillante y con reflejos irisados, que forma el interior de varias conchas y moluscos». $S$ 'il est vrai que la référence aux reflets de la nacre se voit clairement en espagnol lorsqu'il est question de cheveux ou de maquillage, par exemple, il n'en est en revanche guère question si l'on parle des dents ou de la peau, pour lesquelles l'image culturelle correspond à une blancheur éclatante.

22. Dans l'annonce de la crème anti-âge Revitalift parue dans Yo dona (El Mundo, le 23 décembre 2006), l'ambassadrice de L'Oréal est Andie MacDowell, dont on révèle publiquement l'âge (47 ans). Ses soi-disant conseils sont prodigués en tutoyant la lectrice et cliente potentielle: ¡Sé firme contra tus arrugas! Cette pratique du tutoiement se retrouve dans l'annonce de la crème Age re-perfect Procalcium Nuit conçue pour les femmes âgées de plus de soixante ans et dont l'exemple se trouve être Jane Fonda (69 ans). Le message que le publicitaire a choisi de faire passer serait donc le suivant: il n'y a pas de femmes âgées, car grâce à L'Oréal, même une femme de plus de 69 ans ne fait pas son âge. Cela expliquerait en partie l'emploi du tutoiement dans la version espagnole de l'annonce.

23. Le raisonnement naturel chez l'homme (l'enthymème), dont la publicité fait un large usage pour ses fins mercantiles, fonctionne à partir de prémisses fondées sur l'eikôs. Nous renvoyons en ce sens à Vega y Vega (2000) qui explique que «Ces deux concepts fréquent et opinion commune constituent, en gros, l'essentiel de la conception aristotélicienne de la vraisemblance (eikôs). Ils en composent ce qu'on pourrait nommer le "monde [du] probable", celui qui est le plus constant, le plus habituel, le plus conventionnel. Il fournit donc les matériaux (topoï) pour les prémisses, avec lesquelles on va élaborer les enthymèmes qui, à leur tour, vont produire la conviction, la persuasion, la croyance» (Vega y Vega 2000: 106).

\section{RÉFÉRENCES}

Adam, Jean-Michel et Bonhomme, Marc (1997): L'Argumentation publicitaire. Rhétorique de l'éloge et de la persuasion. Paris: Nathan.

Aristote (trad. 1991) : Rhétorique. Livres I, II et III. Paris: Gallimard.

Barthes, Roland (1964/1993): Rhétorique de l'image. In: Roland Barthes, dir. Euvres complètes. Paris: Seuil, 1417-1429.

Benetton, Luciano (2006): Les arts décoratifs. Site officiel. Publicité, consulté le 8 mai 2006, $<$ http://www.lesartsdecoratifs.fr/francais/publicite/collections-97/l-univers-de-la-publicite/marques-et-personnages/benetton/histoire-de-la-publicite-1884>.

Boivineau, Roger (1972): L'a.b.c. de l'adaptation publicitaire. Meta. 17(1):5-28.

Bonнomme, Marc et Rinn, Michael (1997): Peut-on traduire la publicité? L'exemple des annonces romandes et alémaniques. Communication et pragmatique interculturelles, Bulletin suisse de linguistique appliquée. 65:9-24. 
Charaudeau, Patrick (2004): La langue entre identité culturelle et identité discursive. In: José M. Oliver Frade, dir. Isla abierta. Estudios franceses en memoria de Alejandro Cioranescu. Tome I. La Laguna: Universidad de La Laguna, 31-44.

Cordonnier, Jean-Louis (1995): Traduction et culture. Paris: Hatier/Didier.

Cordonnier, Jean-Louis (2002): Aspects culturels de la traduction: quelques notions clés. Meta. 47(1):38-50.

De Pedro, Raquel (1996): Beyond the Words: The translation of Television Adverts. Babel. 42(1):27-43.

Diccionario de la Lengua Española. (2005): Madrid: Espasa-Calpe.

Grize, Jean-Blaise (1981): L’argumentation: explication ou séduction. In: Oswald Ducrot, dir. L'argumentation. Lyon: Presses Universitaires de Lyon, 29-40.

GUIDÈRE, Marc (2001): Translation practices in international advertising. Translation Journal. 5(1), consulté le 15 juin 2006, <http://www.proz.com/translation-articles/articles/276/1/ Translation-Practices-in-International-Advertising $>$.

Kerbrat-Orecchioni, Catherine (1977): La Connotation. Lyon: Presses Universitaires de Lyon.

Ladmiral, Jean-René (1994): Traduire: Théorèmes pour la traduction. Paris: Gallimard.

LAdmiral, Jean-René (2004): Lever de rideau théorique: quelques esquisses conceptuelles. Palimpsestes. 16:15-30.

Lederer, Marianne (1994): La traduction aujourd'hui. Le modèle interprétatif. Paris: Hachette.

Ministère français de l'agriculture et de la pêche (2007): L'appellation d'origine, consulté le 10 mars 2009, <http://agriculture.gouv.fr/sections/thematiques/alimentation/signes-dequalite/les-signes-d-identification-de-la-qualite-et-de-l-origine/l-appellation-d-origine>.

NidA, Eugène A. (1964): Toward a Science of Translating. Leiden: Brill.

Perelman, Chaïm (1977): L'empire rhétorique. Rhétorique et argumentation. Paris: Vrin.

Perelman, Chaïm et Olbrechts-Tyteca, Lucie (1992): Traité de l'argumentation. Bruxelles: Université Libre de Bruxelles.

Platon (1974): Gorgias, Ménon. Paris: Les Belles Lettres.

Preti, Giulio (1974): Retorica et logica: Le due culture. Torino: Einaudi.

Tatilon, Claude (1990): Le texte publicitaire: traduction ou adaptation? Meta. 35(1):243-246.

Vega y Vega, Jorge Juan (2000): L'Enthymème. Histoire et actualité de l'inférence du discours. Lyon: Presses Universitaires de Lyon.

Vinay, Jean-Paul et Darbelnet, Jean-Louis (1977): Stylistique comparée du français et de l'anglais. Méthode de traduction. Édition révisée et corrigée. Paris: Didier.

Zuberoa, Marcos (2007): Así nos transforma el dinero. Muy interesante. 309:36-42. 